\title{
Updated distribution maps of predominant Culex mosquitoes across the Americas
}

\author{
Morgan E. Gorris ${ }^{1 *} \mathbb{D}$, Andrew W. Bartlow², Seth D. Temple ${ }^{3,4}$, Daniel Romero-Alvarez ${ }^{1,5,6}$, Deborah P. Shutt ${ }^{1}$, \\ Jeanne M. Fair ${ }^{2}$, Kimberly A. Kaufeld ${ }^{3}$, Sara Y. Del Valle ${ }^{1}$ and Carrie A. Manore ${ }^{1}$
}

\begin{abstract}
Background: Estimates of the geographical distribution of Culex mosquitoes in the Americas have been limited to state and provincial levels in the United States and Canada and based on data from the 1980s. Since these estimates were made, there have been many more documented observations of mosquitoes and new methods have been developed for species distribution modeling. Moreover, mosquito distributions are affected by environmental conditions, which have changed since the 1980s. This calls for updated estimates of these distributions to understand the risk of emerging and re-emerging mosquito-borne diseases.
\end{abstract}

Methods: We used contemporary mosquito data, environmental drivers, and a machine learning ecological niche model to create updated estimates of the geographical range of seven predominant Culex species across North America and South America: Culex erraticus, Culex nigripalpus, Culex pipiens, Culex quinquefasciatus, Culex restuans, Culex salinarius, and Culex tarsalis.

Results: We found that Culex mosquito species differ in their geographical range. Each Culex species is sensitive to both natural and human-influenced environmental factors, especially climate and land cover type. Some prefer urban environments instead of rural ones, and some are limited to tropical or humid areas. Many are found throughout the Central Plains of the USA.

Conclusions: Our updated contemporary Culex distribution maps may be used to assess mosquito-borne disease risk. It is critical to understand the current geographical distributions of these important disease vectors and the key environmental predictors structuring their distributions not only to assess current risk, but also to understand how they will respond to climate change. Since the environmental predictors structuring the geographical distribution of mosquito species varied, we hypothesize that each species may have a different response to climate change.

Keywords: Mosquitoes, Niche model, Species distribution, Vectors, Mosquito-borne disease, Infectious disease

\section{Background}

Culex mosquitoes, which frequently take blood meals from animals-including humans, are important transmitters of emerging and re-emerging pathogens [1-4]. These mosquitoes are often found in temperate climate zones and are known to be vectors for several

*Correspondence: mgorris@lanl.gov

1 Information Systems and Modeling, Los Alamos National Laboratory, Los Alamos, NM, USA

Full list of author information is available at the end of the article arboviruses, including West Nile virus (WNV), St. Louis encephalitis virus (SLEV), eastern equine encephalitis virus (EEEV), and western equine encephalitis virus (WEEV) (Table 1). For example, WNV is the largest cause of mosquito-borne disease in humans in the United States and is maintained in nature in a wild birdmosquito life-cycle involving a variety of avian hosts and several Culex species. Culex nigripalpus, Culex pipiens, Culex quinquefasciatus, and Culex tarsalis are highly efficient in maintaining and transmitting $\mathrm{WNV}[2,3,5,6]$. Some species transmit parasites as well, such as filarial 
Table 1 Summary of the pathogens that are vectored by the seven focal Culex species and the general regions in which these species are found

\begin{tabular}{|c|c|c|c|}
\hline Species & Pathogen & General region & References \\
\hline Culex erraticus & $\begin{array}{l}\text { EEEV, Venezuelan equine encephalitis virus, WNV, Zika } \\
\text { virus }\end{array}$ & $\begin{array}{l}\text { Southeastern USA, Midwestern USA, Mexico, South } \\
\text { America }\end{array}$ & [92-95] \\
\hline Culex nigripalpus & $\begin{array}{l}\text { EEEV, SLEV, WNV, Zika virus, dog heartworm, avian } \\
\text { malaria }\end{array}$ & Southeastern USA, Mexico, South America & {$[4,96-98]$} \\
\hline Culex pipiens & SLEV, WNV, Zika virus, filarial worms, avian malaria & Mexico, Canada, Midwestern USA, northeast USA & {$[3,96,99]$} \\
\hline Culex quinquefasciatus & SLEV, WEEV, WNV, Zika virus, lymphatic filariasis & Southern USA, Mexico, South America & $\begin{array}{l}{[24,98} \\
100-102]\end{array}$ \\
\hline Culex restuans & SLEV, WNV & Canada, Mexico, eastern USA & {$[3,98,99]$} \\
\hline Culex salinarius & SLEV, WEEV, WNV & Midwestern USA, northeastern USA, southeastern USA & {$[98,99]$} \\
\hline Culex tarsalis & SLEV, WEEV, WNV, Zika virus & Mexico; west of the Mississippi, USA; southeastern USA & $\begin{array}{l}{[96,98,} \\
103]\end{array}$ \\
\hline
\end{tabular}

EEEV Eastern equine encephalitis virus, WNV West Nile virus, SLEV St. Louis encephalitis virus, WEEV western equine encephalitis virus

nematodes and the Plasmodium parasites that cause avian malaria (Table 1).

Disease outbreaks facilitated by Culex species are increasing in frequency, and these mosquitoes will continue to be important vectors for emergent and reemerging diseases [7-9]. For example, outbreaks of EEEV occurred in multiple states within the USA in summer 2019, which resulted in 34 infections and 11 deaths [10]. Spillover events of EEEV into dead end hosts, such as humans and horses, occur unpredictably and are mediated by Culex mosquitoes [11]. Outbreaks of EEEV and similar diseases are difficult to predict because of the complex interactions between reservoir hosts, environmental conditions, human activity, and mosquito distributions [12]. Understanding the spatial distribution of Culex species that are important for the transmission of EEEV and other diseases can provide insight on areas prone to disease risk.

Our current understanding of the geographical distributions of Culex species in North America and South America is mainly limited to studies carried out at state and provincial levels in the USA and Canada that were published in the early 1980s [13]. Though the distribution maps have been updated recently with new species and additional presence data, the estimates of the spatial extent of Culex species have generally remained the same [14]. Furthermore, climate and environmental conditions have continued to change in the years since these updates, including further warming of surface air temperatures and shifts in precipitation [15].

Significant changes to climate are occurring around the world, which influence where mosquitoes can survive. Areas are becoming warmer and wetter or drier [15], causing the geographic distributions of mosquitoes to expand, contract, and/or shift in response [12, 16-18]. In the near future, it is estimated that North America,
Central America, and South America will experience warmer temperatures and more variable precipitation patterns $[19,20]$. There will also likely be an increase in the number of extreme weather events, leading to increased flooding in some areas and long-term droughts in others [19]. Increasing temperatures result in quicker life-cycles of mosquitoes, which allow them to colonize new areas within their temperature limits [21, 22]. Changes to precipitation patterns will promote suitable habitats (e.g., standing water) for them in some areas, but decrease suitability where habitats become drier [21].

There is already evidence that several Culex species have experienced recent shifts in their geographical ranges, with some species expanding northward into Canada. For example, Culex salinarius and Culex erraticus have been consistently trapped in Ontario, Canada in recent years [23]. This finding contradicts the distribution maps produced by Darsie and Ward [14], which show $C x$. salinarius and $C x$. erraticus having northernmost ranges in the northern and Midwestern USA states, respectively [14]. Culex pipiens and Cx. quinquefasciatus are both predicted to spread northward, too [17, 24]. In fact, $C x$. pipiens has already been identified in Canada, and it is likely that this species will undergo further range expansion northward [17].

In parallel to expanding mosquito species distributions, several mosquito-borne viruses are expanding as well, impacting novel and susceptible human populations [9, 18, 25]. For example, since 2014, specific strains of SLEV thought to be restricted to Argentina have been reported in Arizona and California [9, 26]. There has also been an increased risk for WNV in Canada, both in urban and rural areas [27]. Knowing which mosquito species and their associated pathogens are present in an area may assist physicians in providing accurate disease diagnoses and timely, appropriate medical treatments, as well as 
help mosquito control districts identify and control these species [28].

Recent developments in mathematical ecology offer statistical methods to model geographic ranges for organisms when presence records are available. One such method is Maxent [29, 30]. Maxent estimates occurrence intensities by relating species presence data and background locations to environmental predictors in the context of a generalized linear model [31]. It aims to learn the environmental conditions that are suitable for species presence based on the environmental conditions at the recorded presence data points. Because systematically sampling large areas for species presence is difficult, this statistical method provides a tractable and practical way to determine species distributions by making efficient use of species presence data and remotely sensed environmental covariates. This approach also affords information to researchers and public officials because it proposes environmental conditions important to the distribution of the species.

The goal of our study was to use contemporary mosquito and environmental data to update species distribution maps of seven Culex species found in North America and South America: Culex erraticus, Culex nigripalpus, Culex pipiens, Culex quinquefasciatus, Culex restuans, Culex salinarius, and Culex tarsalis. To achieve this, we first surveyed the literature for articles describing environmental conditions that may be important for these seven Culex species. This literature survey highlighted the variables that promote or inhibit Culex species' persistence and provided information on the geographical locations where these species reside. We gathered environmental datasets for use in model development, such as temperature, humidity, and land cover. Then, we used mosquito presence data from several data repositories and the environmental datasets in Maxent to estimate the contemporary distribution of these seven Culex species, examining the environmental variables important for structuring the patterns of habitat suitability. Our contemporary Culex distribution maps may be used to assess mosquito-borne disease risk and forecast future threats from emerging diseases carried by these Culex species.

\section{Methods}

\section{Mosquito presence data}

We focused our analyses on seven predominant Culex species found in North America and South America: $C x$. erraticus, $C x$. nigripalpus, $C x$. pipiens, $C x$. quinquefasciatus, $C x$. restuans, $C x$. salinarius, and $C x$. tarsalis. We chose these species because of the number of diseases they can transmit, the extent of their presence, and the data available. We gathered mosquito presence data from 1990 to 2020 from three data repositories: VectorBase
[32], VectorMap (http://vectormap.si.edu; collected 22 September 2020), and the National Ecological Observatory Network ([33]; collected 4 May 2020), and from surveys done by two public health departments-Public Health of Ontario (2002-2017), and Washington State Department of Health (2008-2014). We included observations that had two decimal point precision $(\sim 1 \mathrm{~km})$ in their latitude and longitude. To account for the clustering of occurrence points and sampling bias, but also maintain the necessary environmental gradient to capture a reliable signal of habitat suitability, we filtered our mosquito presence data using the package spThin in $\mathrm{R}$ using a $30-\mathrm{km}$ radial buffer, so that only one species presence point was used within that buffer area [34, 35]. After filtering, we had between 79 and 300 presence data points per species (Additional file 1: Table S1). As a sensitivity analysis during Maxent model development, we also tried 50-, 75-, and 100-km radial buffers, and the results remained stable (data not shown).

\section{Climate and environmental data}

We reviewed the literature on the seven Culex species to find articles describing important climate and environmental drivers for mosquito presence. Using our literature review as a basis for variable selection, we gathered climate and environmental data that we found to be relevant for providing mosquito habitat and supporting the mosquito life-cycle. We used data from two different sources: MERRAclim [36] and EarthEnv [37-39]. These data sources provide remotely sensed variables relating to climate, land cover, terrain, and biodiversity at resolutions of approximately 1 and $5 \mathrm{~km}$.

We used the most contemporary available MERRAclim data, which was averaged from 2000 to 2009 [36]. We included five measures of temperature: annual mean temperature, mean diurnal range in temperature, the maximum temperature of the warmest month, the minimum temperature of the coldest month, and the annual range in temperature. We included three measures of atmospheric moisture: annual mean specific humidity, specific humidity of the most humid month, and specific humidity of the least humid month. Each variable was available at $2.5-\operatorname{arcmin}(\sim 4 \mathrm{~km})$ resolution.

Each EarthEnv dataset spanned its own time frame based on the product. We included one measure of habitat heterogeneity derived from vegetation, called the evenness of the enhanced vegetation index (EVI), which is bounded from zero to one. This dataset was available at $30-\operatorname{arcsec}(\sim 1 \mathrm{~km})$ resolution and based on the Moderate Resolution Imaging Spectroradiometer (MODIS) EVI product (MOD13Q1 version 5; 250-m resolution) from 2001 to 2005 [39]. We included 12 different land cover classes derived from multiple datasets [GlobCover 
(2005-2006; v2.2), the MODIS land cover product (MCD12Q1; v051), GLC2000 (global product; v1.1), and DISCover (GLCC; v2)]. These classifications are available at 30-arcsec $(\sim 1 \mathrm{~km})$ resolution: evergreen/deciduous needleleaf trees, evergreen broadleaf trees, deciduous broadleaf trees, mixed/other trees, shrubs, herbaceous vegetation, cultivated and managed vegetation, regularly flooded vegetation, urban/built-up, snow/ice, barren, and open water [38]. Lastly, we included four measures of topography based upon the global 250-m GMTED2010 and near-global 90-m SRTM4.1dev products: elevation, slope, roughness, and a terrain ruggedness index [37].

We aggregated each dataset from its native resolution to match the $30-\mathrm{km}$ resolution of our filtered presence data. Matching these resolutions ensures that only one presence data point falls within a single environmental grid. As a result, Maxent analyzes the occurrence record and the environmental grid as a coordinate pair. We visually inspected this aggregated data to verify that coarsening did not noticeably alter the geographic pattern of the environmental variables. We included all the environmental variables in each model in order to infer the contribution of different predictors for each species.

\section{Maxent modeling}

We used the machine learning, maximum entropy model Maxent (maxnet package, version 0.1.2, based on Maxent version $3.4 .0 ;[29,30]$ ) to create species distribution maps of the seven predominant Culex species. We used the $30-\mathrm{km}$ filtered presence data and $30-\mathrm{km}$ climate and environmental data as input to the model. To select the environmental training area for Maxent (i.e., the study area $M$ as defined by Soberon and Peterson [40]), we created a buffer distance for each species based on its presence data and our assumption that mosquito species can readily disperse given their short life-cycles and flight ranges [41-43]. We calculated the buffer length by first computing the centroid of each species' presence data points and then computing the median distance from each presence point to the centroid [44]. We applied this median distance as the radius for a buffer around each presence point. This procedure creates an environmental training area unique to each species (Additional file 1: Figs. S1, S2). From this environmental training area, we initiated Maxent to randomly sample 10,000 background points and trained the models using cross-validation with ten random $\mathrm{k}$-folds of the presence data points. We considered three different feature classes to model relationships between environmental predictors and presence data: linear (L); linear and quadratic (LQ); and linear, quadratic, and hinge (LQH) [45]. We also tested a suite of regularization parameters for model fit, including $0.5,1$,
$2,5,10,20$. Larger regularization parameters encourage models with fewer covariates, lowering overfitting [46].

We selected a best model for each species based on criteria calculated from the ENMevaluate package ([47]; version 0.3.1). Maxent practitioners commonly make model selections based on the corrected Akaike information criterion (AICc), but this approach suffers from poor geographic prediction accuracy [48]. On the other hand, models built by optimizing prediction accuracy may fail to detect environmental features of biological importance [49]. In acknowledgment of these shortcomings, we implemented a custom procedure to select our final models. First, we subsetted the models to only consider the half of the models with the lowest absolute bias in omission rates. For the omission rate, we used the percent of testing presences that had a predicted suitability of less than the 10th quantile of the predicted suitability among the training presences (avg.test.or10pct; [50]). Second, we further subsetted the models to include those that passed a threshold based on the difference between the training and testing area under the curve (AUC) averaged across the ten $\mathrm{k}$-fold bins (avg.diff.AUC). That threshold was set as the median average AUC difference among the fitted models. After these two steps, we selected the model with the lowest AICc. This ad hoc procedure balances prediction accuracy in geographic space and model explainability in ecological space.

After selecting the best Maxent model for each species (i.e., feature classes and regularization parameters; Additional file 1: Table S2), we obtained ten model output replicates to assess the variability in our predictions $[35,51]$. For the ten replicates, we bootstrapped our mosquito presence data to use $80 \%$ of the available presence data. We used the difference between the maximum and minimum habitat suitability output among the ten bootstrapped replicates (i.e., the range) to show areas of higher or lower uncertainty in our models [51, 52].

We were able to analyze the role of each environmental variable in the Maxent models using the mean permutation importance and mean percent variable contribution calculated across the ten bootstrapped models [53]. The permutation importance describes the most important factors in determining the habitat suitability. For each environmental variable in turn, its values are randomly permuted, the model performance is recalculated based on the permuted training data, and the change in model performance is recorded. These differences are then normalized to percentages, where a higher percentage means the model depends heavily on that variable [54]. Maxent also calculates percent contributions from each variable, but these are heuristically defined based on the path the algorithm takes to a locally optimal solution. Although they should be interpreted with caution, together the 
percent variable contribution and variable permutation importance highlight important environmental predictors.

After training the Maxent models with species-specific background environments (Additional file 1: Figs. S1, S2), we extrapolated each bootstrapped model to create mean species distribution maps across the continents in which we expected the mosquito species to occur. We analyzed $C x$. pipiens, $C x$. tarsalis, $C x$. salinarius, and $C x$. restuans for North America and Cx. erraticus, $C x$. quinquefasciatus, and Cx. nigripalpus for North America and South America. For a large number of background points, a Maxent model can be viewed as a Poisson regression model and its outputs can be interpreted as occurrence intensities from a spatial point process [31]. This equivalency motivates the complementary log-log transformation applied to these model intensities as probabilities of presence, subject to some assumptions about the sampling scheme [30]. We used these transformed model outputs because they are normalized to the $[0,1]$ range, but we caution against the aforementioned interpretation; instead, we describe the outputs as "relative habitat suitability" with "low" suitability marked at 0 , "medium" suitability marked at 0.5 , and "high" suitability marked at 1 . These measures of relative suitability are unique for each Culex species based on the unique Maxent model development.

We also created maps to identify any areas with novel climate or environmental conditions outside of the background environmental training data, so that decisions can be weighed appropriately where the model is extrapolated. To do this, we calculated the species-specific ranges of background environmental data variables and highlighted any geographical areas on the map in which at least one variable was outside of these ranges.

\section{Results}

\section{Environmental drivers of Culex mosquito distributions}

We surveyed the literature to identify the geographic region in which each Culex species has been documented and which diseases each species may transmit (Table 1). While all seven species have been documented in North America, only three of the seven have been documented in South America.

We also identified environmental drivers that may be important in structuring the geographical distribution of the seven Culex species of interest (Additional file 1: Table S3) [16, 17, 42, 55-85]. Each Culex species is sensitive to both natural and human-influenced environmental factors, especially land cover and vegetation type. For example, some Culex species were less likely to be found in urban areas, such as $C x$. tarsalis [55] and Cx. nigripalpus [56], whereas others were more likely to be found in these, such as $C x$. pipiens $[55,57,58]$ and $C x$. quinquefasciatus [59]. To account for these differences, we used 12 different land cover types as predictors in our species distribution models, including an "urban/built up" layer. We found evidence that temperature and measures of environmental moisture are important drivers for most Culex species (Additional file 1: Table S3). For this reason, we incorporated both temperature and humidity variables as predictors in our models.

\section{Species distribution maps of Culex mosquitoes}

We gathered contemporary mosquito presence data for the Culex mosquito species in North America (Fig. 1) and North America and South America (Fig. 2) for use in the Maxent models. We tried to find presence data to cover the hypothesized geographical range within the USA and Canada highlighted by Darsie and Ward [14]. Though the study of Darsie and Ward [14] was limited to estimating the range within the USA and Canada, many of the presence data points fell within the estimated range. All seven species also had contemporary presence points further north than depicted by Darsie and Ward [14].

The feature classes and regularization parameters varied among the species for the best Maxent model configuration (Additional file 1: Table S2). We also reported measures of prediction accuracy in geographic space (omission rates and AUC) and model explainability (AICc). After selecting the final model configuration and running the bootstrapped models, the resulting mean species distribution maps showed variability in the species ranges between all seven species, both in North America (Fig. 3) and North America and South America (Fig. 4). In North America, Cx. pipiens had the most disparate habitat suitability surrounding urban areas, whereas $C x$. tarsalis had the most widespread suitability across the temperate and subtropical regions of North America, throughout both urban and rural areas. The geographical ranges of $C x$. restuans and $C x$. salinarius were more limited to the eastern and Midwestern USA and the Central Plains of Canada, with the range for $C x$. salinarius dropping further south into Central America and the Caribbean. In North America and South America, $C x$. erraticus and $C x$. quinquefasciatus had a larger range of habitat suitability compared to $C x$. nigripalpus, which was more limited to the tropics and subtropics. In North America, $C x$. erraticus showed a similar pattern of habitat suitability to $C x$. salinarius.

The geographical range of $C x$. pipiens was largely driven by the urban/built-up land cover variable, which was also an important driver for the distributions of $C x$. salinarius, $C x$. tarsalis, and $C x$. quinquefasciatus (Table 2; Additional file 1: Table S4). Measures of temperature were important drivers for five of the Culex 

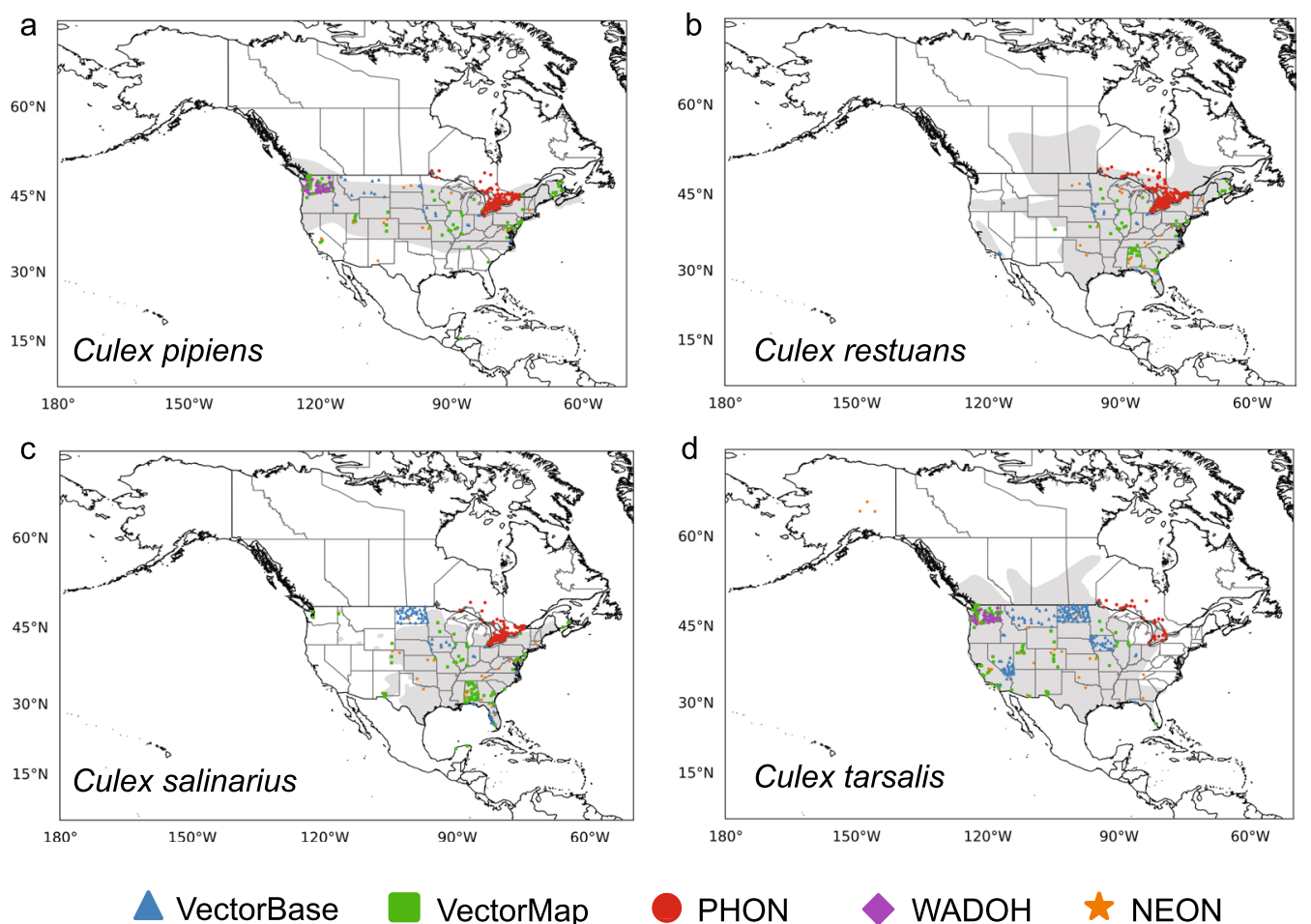

Fig. 1 The presence data points used in Maxent model development from several data repositories for a Culex pipiens, b Culex restuans, c Culex salinarius, and $\mathbf{d}$ Culex tarsalis. The estimated distribution of each species within the USA and Canada is shaded in gray (from Darsie et al. [14]). PHON Public Health of Ontario, WADOH Washington State Department of Health, NEON National Ecological Observatory Network
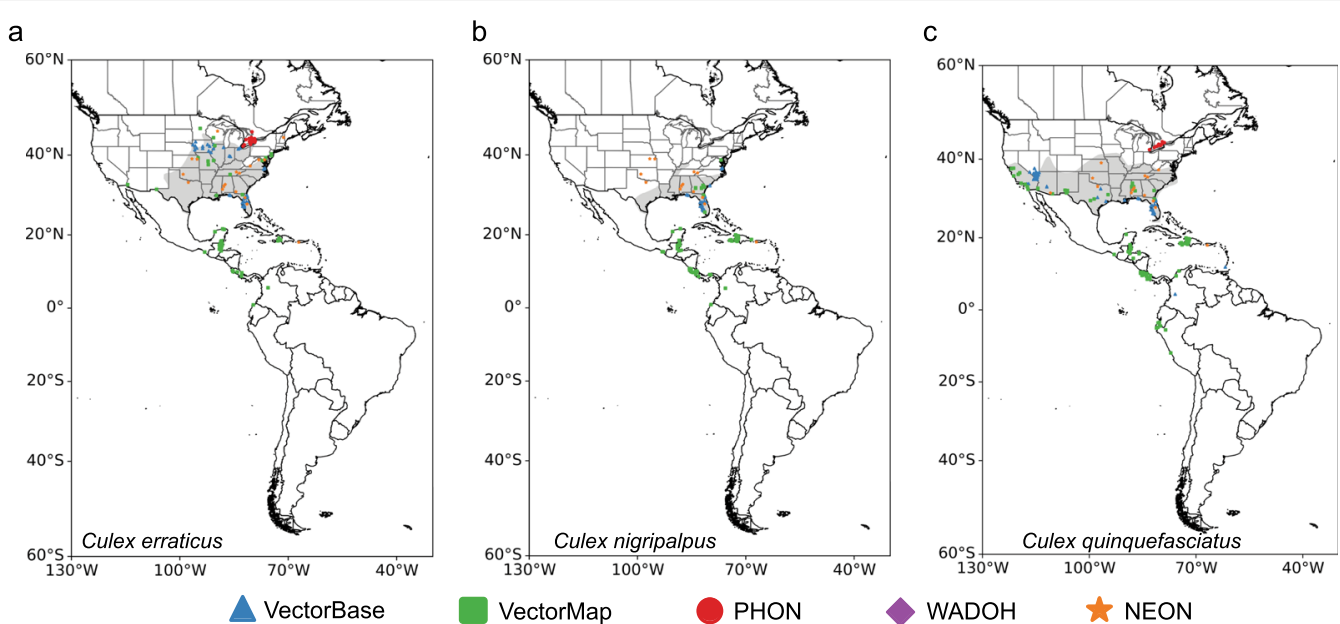

Fig. 2 The presence data points used in Maxent model development from several data repositories for a Culex erraticus, b Culex nigripalpus, and c Culex quinquefasciatus. The estimated distribution of each species within the USA and Canada is shaded in gray (from Darsie et al. [14]). For abbreviations, see Fig. 1

species, while not as important for characterizing the distributions of $C x$. nigripalpus and $C x$. salinarius. The distributions of all species besides $C x$. quinquefasciatus were driven by measures of specific humidity. Cultivated and managed vegetation was another land cover variable that was important for several species, including $C x$. erraticus, Cx. pipiens, Cx. salinarius, and Cx. tarsalisall of which are noted as predominant mosquitoes in the agricultural plains of the USA. Taken in combination, the percent variable contribution (Additional file 1: Table S4) 

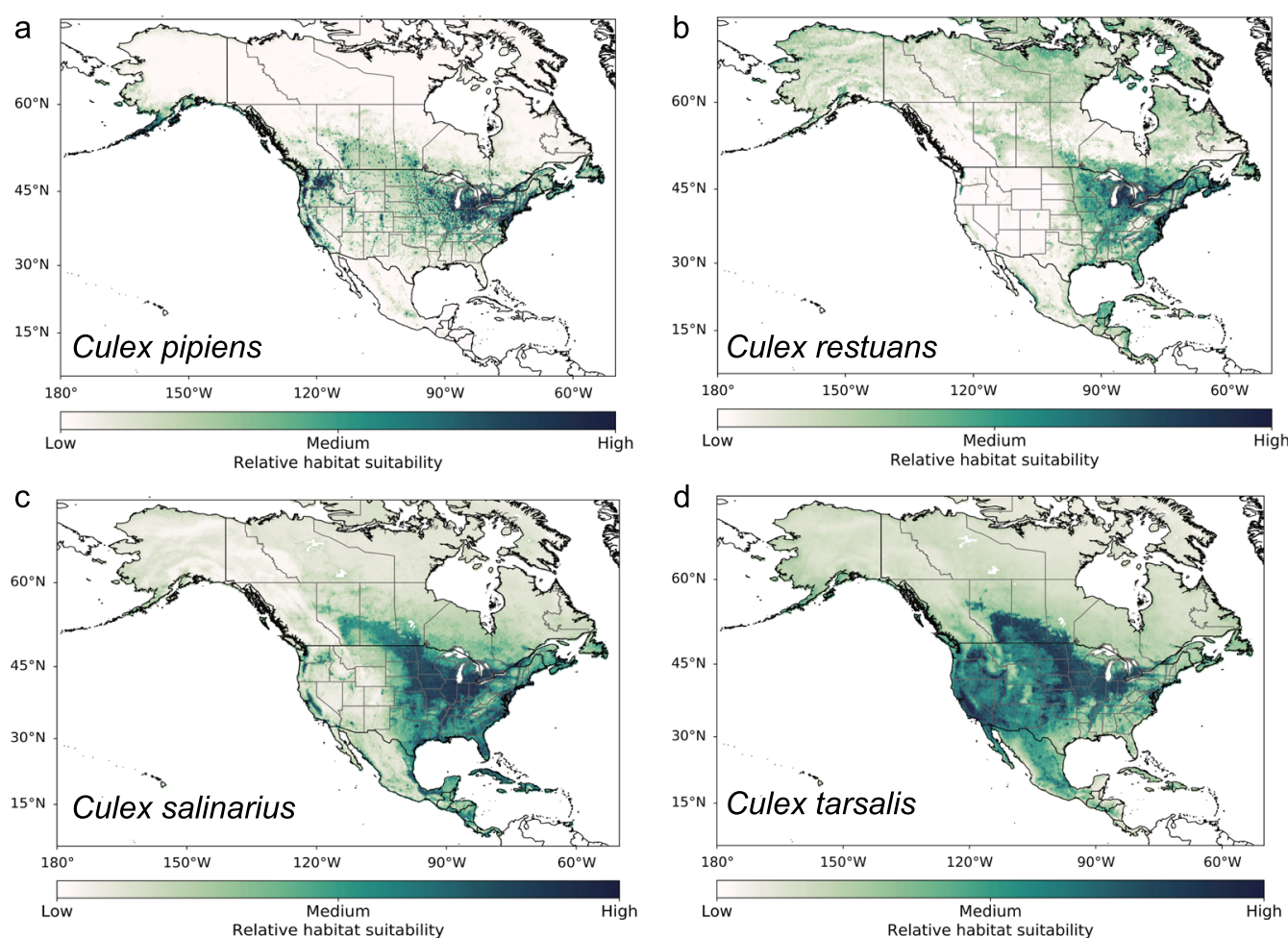

Fig. 3 Mean geographical distribution maps averaged across the ten bootstrapped models for predominant Culex species in North America, including a Culex pipiens, b Culex restuans, c Culex salinarius, and $\mathbf{d}$ Culex tarsalis. The relative habitat suitability is unique to each species based on the Maxent model development
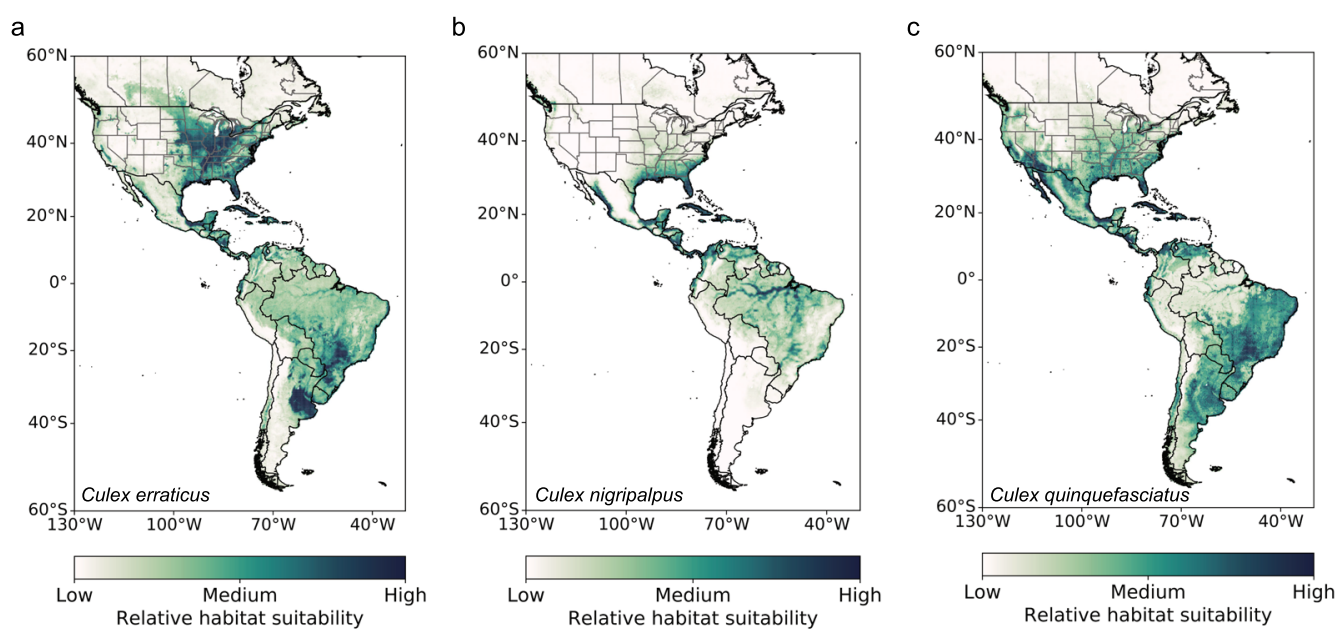

Fig. 4 Mean geographical distribution maps averaged across the ten bootstrapped models for predominant Culex species in North America and South America, including a Culex erraticus, b Culex nigripalpus, and c Culex quinquefasciatus. The relative habitat suitability is unique to each species based on the Maxent model development

and variable permutation importance (Table 2) highlight particularly important variables in structuring habitat suitability for different Culex mosquito species.
Although each model was developed from a unique set of background environmental training data, we extrapolated the models to all of North America and/or South 
Table 2 Percent permutation importance of each environmental variable from Maxent models for each Culex species

\begin{tabular}{|c|c|c|c|c|c|c|c|}
\hline Environmental variable & Culexpipiens & Culex restuans & Culex salinarius & Culextarsalis & Culex erraticus & Culex nigripalpus & $\begin{array}{l}\text { Culex } \\
\text { quinquefasciatus }\end{array}$ \\
\hline \multicolumn{8}{|l|}{ Climate } \\
\hline Annual mean temp. & 26.7 & 4.8 & 6.5 & 6.6 & 0.0 & 1.3 & 2.9 \\
\hline Temp. annual range & 0.3 & 5.4 & 0.0 & 0.0 & 1.6 & 0.0 & 0.0 \\
\hline Mean diurnal temp. range & 1.1 & 1.8 & 0.0 & 0.0 & 0.0 & 0.0 & 18.4 \\
\hline $\begin{array}{l}\text { Maximum temp. in the warmest } \\
\text { month }\end{array}$ & 0.0 & 9.0 & 0.0 & 9.5 & 0.0 & 0.3 & 8.9 \\
\hline $\begin{array}{l}\text { Minimum temp. in the coldest } \\
\text { month }\end{array}$ & 12.5 & 0.4 & 2.3 & 8.4 & 18.7 & 0.0 & 15.1 \\
\hline Annual mean specific humidity & 3.3 & 13.5 & 12.6 & 3.8 & 0.0 & 0.0 & 0.0 \\
\hline $\begin{array}{l}\text { Specific humidity in the most } \\
\text { humid month }\end{array}$ & 10.8 & 1.1 & 1.7 & 30.0 & 18.4 & 60.3 & 2.8 \\
\hline $\begin{array}{l}\text { Specific humidity in the least } \\
\text { humid month }\end{array}$ & 0.5 & 14.6 & 0.0 & 0.0 & 3.4 & 7.4 & 1.4 \\
\hline \multicolumn{8}{|l|}{ Land cover } \\
\hline $\begin{array}{l}\text { Evergreen/deciduous needleleaf } \\
\text { trees }\end{array}$ & 0.1 & 9.2 & 0.1 & 0.2 & 0.4 & 3.3 & 1.0 \\
\hline Evergreen broadleaf trees & 0.0 & 0.7 & 0.0 & 0.0 & 1.1 & 0.4 & 13.3 \\
\hline Deciduous broadleaf trees & 0.2 & 2.0 & 0.3 & 0.8 & 0.1 & 1.5 & 0.4 \\
\hline Mixed/other trees & 0.3 & 1.8 & 0.0 & 0.0 & 12.1 & 0.5 & 1.8 \\
\hline Shrubs & 0.0 & 1.9 & 2.4 & 0.0 & 2.1 & 0.7 & 4.9 \\
\hline Herbaceous vegetation & 0.1 & 12.1 & 0.8 & 0.1 & 10.1 & 0.5 & 2.0 \\
\hline $\begin{array}{l}\text { Cultivated and managed vegeta- } \\
\text { tion }\end{array}$ & 12.3 & 2.0 & 30.2 & 27.2 & 13.8 & 1.6 & 3.2 \\
\hline Regularly flooded vegetation & 0.0 & 3.3 & 0.0 & 0.0 & 0.0 & 0.0 & 0.0 \\
\hline Urban/built-up & 25.6 & 1.2 & 21.9 & 12.4 & 5.7 & 0.8 & 14.1 \\
\hline Snow/ice & 0.0 & 0.0 & 0.0 & 0.5 & 0.0 & $<0.1$ & 0.0 \\
\hline Barren & 0.0 & $<0.1$ & 0.0 & 0.0 & 0.0 & 0.0 & 0.5 \\
\hline Open water & 0.4 & 1.6 & 0.9 & 0.0 & 0.4 & 0.2 & 0.6 \\
\hline \multicolumn{8}{|l|}{ Habitat } \\
\hline Evenness of EVI & 0.1 & 7.5 & 0.0 & 0.0 & 3.7 & 2.9 & 0.0 \\
\hline \multicolumn{8}{|l|}{ Topography } \\
\hline Elevation & 5.7 & 0.7 & 11.9 & 0.5 & 8.4 & 9.6 & 5.9 \\
\hline Roughness index & 0.0 & 0.0 & 0.0 & 0.0 & 0.0 & 0.0 & 0.0 \\
\hline Slope & 0.0 & 5.3 & 8.4 & 0.0 & 0.0 & 3.1 & 2.5 \\
\hline Terrain ruggedness index & 0.0 & 0.0 & 0.0 & 0.0 & 0.0 & 5.5 & 0.1 \\
\hline
\end{tabular}

Temp. Temperature, EVI enhanced vegetation index

America. By doing so, in some areas the models are applied to novel climate or environmental conditions. We created maps to identify these areas (Additional file 1: Figs. S3, S4). If using the map for decision support, these areas should be addressed with caution. These areas mostly include northern boreal and arctic regions, some tropical regions, areas with high elevation, or areas with exceptionally dry conditions.

The range in habitat suitability across the bootstrapped models was low for $C x$. pipiens, $C x$. salinarius, and $C x$. tarsalis, displaying confidence in the model output across North America (Additional file 1: Fig.
S5). The suitability range for $C x$. restuans was highly variable in Central America, so caution should be used when interpreting the results for that region. This area was outside the background environmental data used to train the Maxent model for Cx. restuans and coincides with areas where the model was applied to novel environmental conditions (Additional file 1: Figs. S1, S4). The ranges for $C x$. erraticus, $C x$. nigripalpus, and $C x$. quinquefasciatus all have relatively higher levels of uncertainty, especially across South America (Additional file 1: Fig. S6). 


\section{Discussion}

We used contemporary mosquito data, environmental drivers, and an ecological niche model to create updated estimates of the geographical range of seven predominant Culex species in North America and South America. We found that the geographical range varied across the seven species, but measures of temperature, humidity, urban/ built-up land class, and cultivated and managed vegetation were especially important environmental drivers structuring the spatial distribution of these species. The geographical distributions highlighted known preferences of the different species; for example, the range of $C x$. pipiens indicated that urban environments were more favorable for this species, whereas the range of $C x$. tarsalis indicated that the environments throughout the Great Plains of the USA, the Central Valley of California, and other agricultural areas, were most favorable for this species. Urban/build-up land cover was an important variable for structuring the spatial distribution of $C x$. pipiens, $C x$. quinquefasciatus, $C x$. salinarius, and $C x$. tarsalis; however, the complexities of the Maxent model structure make it challenging to identify the relationship between environmental drivers and the presence of these mosquitoes since there are numerous parameters and model coefficients that could be compensating for other similar drivers.

Our Culex species distribution maps leverage contemporary mosquito presence data from several sources to update the hypothesized distribution for seven important disease vectors. The majority of these data sources classify which Culex species is present by using morphology, which is prone to error [86]. The Cx. pipiens species complex consists of several morphologically similar species that are difficult to visually identify [87], which includes $C x$. pipiens and $C x$. quinquefasciatus within our study. In fact, some databases allow generic species identification entries of "Culex pipiens morphological group", which we did not include in our Maxent models to reduce uncertainty. However, one of our data sources, VectorBase, includes Culex species identified from genomics data, eliminating some of the error from misidentification. Creating a species distribution map using genomics data alone would increase the precision of the estimated geographical ranges; currently, this approach is not feasible given the limited number of presence data from sequencing. Identifying the correct species is important since they can play different roles in disease transmission. For example, for WNV, while $C x$. pipiens primarily feeds on birds, Cx. quinquefasciatus feeds on both birds and mammals, which may act as a bridge to amplify the transmission of WNV between avian and mammalian hosts [88]. Using genomics data can also highlight areas where there are hybrids, which can alter disease transmission dynamics [89]. With so many agencies in the USA collecting mosquito data for both abatement and control measures and scientific research, it would be transformative to have a central data repository for mosquito species and disease incidence data.

Our maps may be suitable for estimating where a species could readily spread if introduced to a new area, targeting potential environmental sampling for a species, or analyzing potential disease pathways in response to an emergent mosquito-borne disease. However, there are several considerations when analyzing the maps. First, the majority of our presence data points were limited to North America, which could potentially generate more uncertainty for the hypothesized distributions in South America. Second, these distribution maps are a measure of relative habitat suitability rather than the true presence of the organism. Other factors, such as interannual climate conditions, land use, and species migration, govern the presence of the organism within the potential distribution [90]. Lastly, due to the complex nature of Maxent models, they may behave in unexpected ways when extrapolated to novel climate conditions. It is important to recognize areas with novel climate conditions relative to the background environmental data; we note that these areas should be flagged as more uncertain when using these maps for applications.

Our distribution maps corroborate niche models recently developed for Culex species using different data sources and modeling techniques at other spatial scales. A global study of the distribution of $C x$. quinquefasciatus using more presence data points in Central America and South America but fewer in the USA and Canada shows a similar pattern of $C x$. quinquefasciatus limited to the southern part of the USA and suitable habitat throughout Central America and much of South America [24]. However, our map also shows a low level of habitat suitability throughout much of the Amazon rainforest. A study using logistic regression models of $C x$. pipiens in Canada shows similar results of the geographical range limited to the extreme southeastern portion of Canada, including southern Ontario, Quebec, and Nova Scotia [17]. Our maps are limited by a lower spatial resolution to encompass a greater physical distance, so we cannot capture high resolution nuances in habitat suitability for a species compared to smaller-scale, high resolution studies. For example, our map highlights St. Johns County, Florida, USA as suitable for both $C x$. nigripalpus and $C x$. quinquefasciatus. However, a county-level species distribution map using Maxent and seropositive records of sentinel chickens as presence data points shows heterogeneity in suitability of habitats for these mosquito species within the county [60]. 
Understanding the contemporary distributions of the predominant Culex mosquitoes is critical, as their distributions will likely shift in response to climate change. This may expose new communities to mosquito-borne diseases, or result in emerging diseases [12]. Maxent models can also be used to project future mosquito distributions in response to climate change scenarios, pending reliable projections of future environmental and climate conditions (e.g., [24]). Warming temperatures may cause the geographical range of mosquito species to expand further north [17, 24]. However, increasing temperatures throughout the tropics and subtropics may begin to push some Culex species above their thermotolerance, causing mortality and making certain habitats or regions unsuitable for them [91]. The exact response to increasing temperatures varies between Culex species; for example, $C x$. quinquefasciatus is likely more adapted to survive higher temperatures, while $C x$. pipiens may be more sensitive to them [42].

\section{Conclusions}

Understanding the geographical distributions of disease vectors, such as mosquitoes, is critical for understanding disease risk. We created updated distribution maps of Culex mosquitoes, which are vectors for numerous diseases, throughout North America and South America using contemporary observation data and a machine learning ecological niche model. Our distribution maps provide insight on the key drivers structuring the spatial distribution of Culex mosquitos. Understanding the distribution of vectors is particularly important when a new disease emerges and rapid assessments need to be made for disease mitigation strategies. These estimates can help identify which communities are most at risk based on the primary disease vectors, and provide decision support regarding where to spray pesticides, and/or where to allocate healthcare resources. Further, a baseline understanding of which environmental conditions structure the geographical distributions of vector species is necessary in order to create projections of disease risk in response to climate change. As temperatures warm, precipitation patterns change, and landscapes shift in response to climate change, the geographical distributions of disease vectors, and therefore the diseases they carry, will also change. Since the key environmental drivers and resultant distribution maps for each Culex species were different, we hypothesize that each species will show a unique response to climate change.

\section{Supplementary Information}

The online version contains supplementary material available at https://doi. org/10.1186/s13071-021-05051-3.

Additional file 1: Table S1. The total number of presence data points for each Culex species used in model development after filtering by the $30-\mathrm{km}$ radial buffer. Table S2. Final model specifications and performance metrics for each species. Table S3. Summary of environmental factors important for each of the seven Culex species, which were obtained from the literature review. Table S4. Percent environmental variable contribution during Maxent model development for each Culex species. Figure S1. Maps of the environmental training area unique to each species used for the Maxent models across North America for a Culex pipiens, b Culex restuans, c Culex salinarius, and $\mathbf{d}$ Culex tarsalis. These were created by buffering the data based on the median distance from each presence data point to the centroid of all presence points. Ten thousand background points are randomly sampled from the shaded environmental training area when running Maxent. Figure S2. Maps of the environmental training area unique to each species used for the Maxent models across North America and South America for a Culex erraticus, b Culex nigripalpus, and c Culex quinquefasciatus. These were created by buffering the data based on the median distance from each presence data point to the centroid of all presence points. Ten thousand background points are randomly sampled from the shaded environmental training area when running Maxent. Figure S3. After extrapolating the Maxent models across North America, areas that are highlighted have novel climate or environmental conditions relative to the background environmental training dataset. This is unique to each species: a Culex pipiens, b Culex restuans, c Culex salinarius, and $\mathbf{d}$ Culex tarsalis. Figure S4. After extrapolating the Maxent models across North America and South America, areas that are highlighted have novel climate or environmental conditions relative to the background environmental training dataset. This is unique to each species: a Culex erraticus, b Culex nigripalpus, and c Culex quinquefasciatus. Figure S5. Maps of the difference between the maximum and minimum suitability output amongst the ten bootstrapped replicates (i.e., the range) to show areas of high or low uncertainty in our models for species in North America: a Culexpipiens, b Culex restuans, c Culex salinarius, and d Culex tarsalis. Figure S6. Maps of the difference between the maximum and minimum suitability output amongst the ten bootstrapped replicates (i.e., the range) to show areas of high or low uncertainty in our models for species in North America and South America: a Culex erraticus, b Culex nigripalpus, and $\mathbf{c}$ Culex quinquefasciatus.

\section{Acknowledgements}

We appreciate and thank the Washington State Department of Health and Public Health of Ontario for sharing their mosquito data. This work is approved for distribution under LA-UR-21-24460. The findings and conclusions in this report are those of the authors and do not necessarily represent the official position of their affiliations, including Los Alamos National Laboratory (LANL). LANL, an affirmative action/equal opportunity employer, is managed by Triad National Security, for the National Nuclear Security Administration of the USA Department of Energy under contract 89233218CNA000001. The funders had no role in the study design, data analysis, decision to publish, or preparation of the manuscript.

\section{Authors' contributions}

MEG, AWB, SDT, and DRA designed the study, collected data, developed the computational framework, and wrote the manuscript. DPS collected and analyzed data and provided input to the manuscript. JMF, KAK, SYD, and CAM were involved with planning, supervised the work, and provided input to the manuscript. All authors read and approved the final manuscript. 


\section{Funding}

MEG gratefully acknowledges support from a director's postdoc fellowship through the Laboratory Directed Research and Development (LDRD) program and the Center for Nonlinear Studies at LANL (Grant No. 36A10C-XX6F. AWB also acknowledges support from a director's postdoc fellowship through the LDRD program at LANL (Grant No. 36A10C-XX9E). This work was supported by several additional LDRD projects through LANL (Grant No. 36A10C-XX0N and Grant No. 36A10C-XX4E). The funding sources played no role in the design of the project, analyses, interpretation of results, or writing of the manuscript.

\section{Availability of data and materials}

The habitat suitability values for each mosquito species across the full spatial domains are available as netCDF files at https://github.com/lanl/culexmaxen tmodels.

\section{Declarations}

Ethics approval and consent to participate

Not applicable.

\section{Consent for publication}

Not applicable.

\section{Competing interests}

The authors declare no competing interests.

\section{Author details}

${ }^{1}$ Information Systems and Modeling, Los Alamos National Laboratory, Los Alamos, NM, USA. ${ }^{2}$ Biosecurity and Public Health, Los Alamos National Laboratory, Los Alamos, NM, USA. ${ }^{3}$ Statistical Sciences, Los Alamos National Laboratory, Los Alamos, NM, USA. ${ }^{4}$ Department of Statistics, University of Washington, Seattle, WA, USA. ${ }^{5}$ Biodiversity Institute and Department of Ecology and Evolutionary Biology, University of Kansas, Lawrence, KS, USA. ${ }^{6}$ OneHealth Research Group, Facultad de Medicina, Universidad de las Américas, Quito, Ecuador.

Received: 7 June 2021 Accepted: 1 October 2021

Published online: 23 October 2021

\section{References}

1. Barba M, Fairbanks EL, Daly J. Equine viral encephalitis: prevalence, impact, and management strategies. Vet Med (Auckl). 2019;10:99-110.

2. Hamer GL, Kitron UD, Goldberg TL, Brawn JD, Loss SR, Ruiz MO, et al. Host selection by Culex pipiens mosquitoes and West Nile virus amplification. Am J Trop Med Hyg. 2009;80:268-78.

3. Kilpatrick AM, Kramer LD, Campbell SR, Alleyne EO, Dobson AP, Daszak P. West Nile virus risk assessment and the bridge vector paradigm. Emerg Infect Dis. 2005; 11:425-9.

4. Vitek CJ, Richards SL, Mores CN, Day JF, Lord CC. Arbovirus transmission by Culex nigripalpus in Florida, 2005. J Med Entomol. 2008;45:483-93.

5. Rueda L, Patel K, Axtell R, Stinner R. Temperature-dependent development and survival rates of Culex quinquefasciatus and Aedes aegypti (Diptera: Culicidae). J Med Entomol. 1990;27:892-8.

6. Turell MJ, Dohm DJ, Sardelis MR, O'Guinn ML, Andreadis TG, Blow JA. An update on the potential of North American mosquitoes (Diptera: Culicidae) to transmit West Nile virus. J Med Entomol. 2005;42:57-62.

7. Camp JV, Nowotny N. The knowns and unknowns of West Nile virus in Europe: what did we learn from the 2018 outbreak? Expert Rev AntiInfect. 2020;18:145-54.

8. Diaz A, Coffey LL, Burkett-Cadena N, Day JF. Reemergence of St. Louis encephalitis virus in the Americas. Emerg Infect Dis. 2018;24:2150-7.

9. Swetnam DM, Stuart JB, Young K, Maharaj PD, Fang Y, Garcia S, et al. Movement of St. Louis encephalitis virus in the western United States, 2014-2018. PLoS Negl Trop Dis. 2020;14:e0008343.

10. Lindsey NP, Martin SW, Staples JE, Fischer M. Notes from the field: multistate outbreak of eastern equine encephalitis virus - United States, 2019. Morb Mortal Wkly Rep. 2020;69:50-1.
11. Morens DM, Folkers GK, Fauci AS. Eastern equine encephalitis virusanother emergent arbovirus in the United States. New Engl J Med. 2019;381:1989-92.

12. Bartlow AW, Manore C, Xu C, Kaufeld KA, Del Valle S, Ziemann A, et al. Forecasting zoonotic infectious disease response to climate change: mosquito vectors and a changing environment. Vet Sci. 2019;6:40.

13. Darsie RF, Ward RA. Identification and geographical distribution of the mosquitoes of North America, north of Mexico. Walter Reed Army Inst Res Wash DC; 1981.

14. Darsie RF, Ward RA. Identification and geographical distribution of the mosquitos of North America, North of Mexico. Gainesville, FL: University Press of Florida; 2005

15. IPCC. Climate Change 2014: Synthesis report. Contribution of Working Groups I, II and III to the Fifth Assessment Report of the Intergovernmental Panel on Climate Change. IPCC, Geneva, Switzerland; 2014 p. 151.

16. Chen C, Jenkins E, Epp T, Waldner C, Curry P, Soos C. Climate change and West Nile virus in a highly endemic region of North America. Int J Environ Res Public Health. 2013;10:3052-71.

17. Hongoh V, Berrang-Ford L, Scott ME, Lindsay LR. Expanding geographical distribution of the mosquito, Culex pipiens, in Canada under climate change. Appl Geogr. 2012;33:53-62.

18. Roth D, Henry B, Mak S, Fraser M, Taylor M, Li M, et al. West Nile virus range expansion into British Columbia. Emerg Infect Dis. 2010;16:1251-8.

19. Magrin GO, Marengo JA, Boulanger JP, Buckeridge MS, Castellanos E, Poveda G, et al. Central and South America. Climate Change 2014: impacts, adaptation, and vulnerability. Part B. Regional aspects. Contribution of Working Group II to the Fifth Assessment Report of the Intergovernmental Panel on Climate Change. Cambridge, UK: Cambridge University Press; 2014. p. 1499-566.

20. Romero-Lankao P, Smith JB, Davidson DJ, Diffenbaugh NS, Kinney PL, Kirshen P, et al. North America. Climate change 2014: Impacts, adaptation, and vulnerability. Part B. Regional aspects. Contribution of Working Group II to the Fifth Assessment Report of the Intergovernmental Panel on Climate Change. Cambridge, UK: Cambridge University Press; 2014. p. 1439-98.

21. Wang J, Ogden NH, Zhu H. The impact of weather conditions on Culex pipiens and Culex restuans (Diptera: Culicidae) abundance: a case study in Peel Region. J Med Entomol. 2011;48:468-75.

22. Yoo E-H, Chen D, Diao C, Russell C. The effects of weather and environmental factors on West Nile virus mosquito abundance in Greater Toronto area. Earth Interact. 2016;20:1-22.

23. Giordano BV, Gasparotto A, Hunter FF. A checklist of the 67 mosquito species of Ontario, Canada. J Am Assoc Mosq Contr. 2015;31:101-3.

24. Samy AM, Elaagip AH, Kenawy MA, Ayres CFJ, Peterson AT, Soliman DE. Climate change influences on the global potential distribution of the mosquito Culex quinquefasciatus, vector of West Nile virus and lymphatic filariasis. PLoS ONE. 2016;11:e0163863.

25. Gibney KB, Robinson S, Mutebi J-P, Hoenig DE, Bernier BJ, Webber L, et al. Eastern equine encephalitis: an emerging arboviral disease threat, Maine, 2009. Vector-Borne Zoonotic Dis. 2011;11:637-9.

26. Auguste AJ, Pybus OG, Carrington CVF. Evolution and dispersal of St. Louis encephalitis virus in the Americas. Infect Genet Evol. 2009:9:709-15.

27. Ludwig A, Zheng H, Vrbova L, Drebot M, Iranpour M, Lindsay L. Increased risk of endemic mosquito-borne diseases in Canada due to climate change. Can Commun Dis Rep. 2019;45:91-7.

28. Khasnis AA, Nettleman MD. Global warming and infectious disease. Arch Med Res. 2005;36:689-96.

29. Phillips SJ, Anderson RP, Schapire RE. Maximum entropy modeling of species geographic distributions. Ecol Model. 2006;190:231-59.

30. Phillips SJ, Anderson RP, Dudík M, Schapire RE, Blair ME. Opening the black box: an open-source release of Maxent. Ecography. 2017;40:887-93.

31. Renner IW, Warton DI. Equivalence of Maxent and Poisson point process models for species distribution modeling in ecology. Biometrics. 2013;69:274-81.

32. Giraldo-Calderón Gl, Emrich SJ, MacCallum RM, Maslen G, Dialynas E, Topalis P, et al. VectorBase: an updated bioinformatics resource for 
invertebrate vectors and other organisms related with human diseases. Nucleic Acids Res. 2015;43:D707-13.

33. National Ecological Observatory Network (NEON). Mosquitoes sampled from $\mathrm{CO}_{2}$ traps. National Ecological Observatory Network (NEON); 2021. Available from: https://data.neonscience.org/data-products/DP1.10043. 001/RELEASE-2021.

34. Aiello-Lammens ME, Boria RA, Radosavljevic A, Vilela B, Anderson RP. spThin: an R package for spatial thinning of species occurrence records for use in ecological niche models. Ecography. 2015;38:541-5.

35. Sillero N, Barbosa AM. Common mistakes in ecological niche models. Int J Geogr Inf Sci. 2021;35:213-26.

36. Vega GC, Pertierra LR, Olalla-Tárraga MÁ. Data from: MERRAclim, a high-resolution global dataset of remotely sensed bioclimatic variables for ecological modelling. Dryad. 2018;Dataset.

37. Amatulli G, Domisch S, Tuanmu M-N, Parmentier B, Ranipeta A, Malczyk J, et al. A suite of global, cross-scale topographic variables for environmental and biodiversity modeling. Sci Data. 2018;5:180040.

38. Tuanmu M-N, Jetz W. A global 1-km consensus land-cover product for biodiversity and ecosystem modelling: consensus land cover. Global Ecol Biogeogr. 2014;23:1031-45.

39. Tuanmu M-N, Jetz W. A global, remote sensing-based characterization of terrestrial habitat heterogeneity for biodiversity and ecosystem modelling: global habitat heterogeneity. Global Ecol Biogeogr. 2015:24:1329-39.

40. Soberon J, Peterson AT. Interpretation of models of fundamental ecological niches and species' distributional areas. Biodiv Inf. 2005;2:1-10.

41. Barve N, Barve V, Jiménez-Valverde A, Lira-Noriega A, Maher SP, Peterson AT, et al. The crucial role of the accessible area in ecological niche modeling and species distribution modeling. Ecol Model. 2011;222:1810-9.

42. Ciota AT, Matacchiero AC, Kilpatrick AM, Kramer LD. The effect of temperature on life history traits of Culex mosquitoes. J Med Entomol. 2014;51:55-62.

43. Sallam M, Michaels S, Riegel C, Pereira R, Zipperer W, Lockaby B, et al. Spatio-temporal distribution of vector-host contact (VHC) ratios and ecological niche modeling of the West Nile virus mosquito vector, Culex quinquefasciatus, in the city of New Orleans, LA, USA. IJERPH. 2017;14:892.

44. Poo-Muñoz DA, Escobar LE, Peterson AT, Astorga F, Organ JF, MedinaVogel G. Galictis cuja (Mammalia): an update of current knowledge and geographic distribution. Iheringia Ser Zool. 2014;104:341-6.

45. Merow C, Smith MJ, Silander JA. A practical guide to MaxEnt for modeling species' distributions: what it does, and why inputs and settings matter. Ecography. 2013;36:1058-69.

46. Radosavljevic A, Anderson RP. Making better Maxent models of species distributions: complexity, overfitting and evaluation. J Biogeogr. 2014;41:629-43.

47. Muscarella R, Galante P, Soley-Guardia M, Boria R, Kass J, Uriarte M. ENMeval: an R package for conducting spatially independent evaluations and estimating optimal model complexity for Maxent ecological niche models. Methods Ecol Evol. 2014;5:1198-205.

48. Velasco JA, González-Salazar C. Akaike information criterion should not be a "test" of geographical prediction accuracy in ecological niche modelling. Ecol Inform. 2019;51:25-32.

49. Warren DL, Matzke NJ, Iglesias TL. Evaluating presence-only species distribution models with discrimination accuracy is uninformative for many applications. J Biogeogr. 2020;47:167-80.

50. Pearson RG, Raxworthy CJ, Nakamura M, Townsend PA. Predicting species distributions from small numbers of occurrence records: a test case using cryptic geckos in Madagascar. J Biogeogr. 2006;34:102-17.

51. Simoes $M$, Romero-Alvarez D, Nuñez-Penichet $C$, Jiménez L, Cobos ME. General theory and good practices in ecological niche modeling: a basic guide. Biodiv Inf. 2020;15:67-8.

52. Romero-Alvarez D, Peterson AT, Salzer JS, Pittiglio C, Shadomy S, Traxler R, et al. Potential distributions of Bacillus anthracis and Bacillus cereus biovar anthracis causing anthrax in Africa. PLoS Negl Trop Dis. 2020;14:e0008131.

53. Cobos ME, Peterson AT, Osorio-Olvera L, Jiménez-García D. An exhaustive analysis of heuristic methods for variable selection in ecologica niche modeling and species distribution modeling. Ecol Inform. 2019;53:100983.

54. Phillips SJ. A brief tutorial on Maxent. AT\&T Res. 2005:190:231-59.

55. DeGroote JP, Sugumaran R, Brend SM, Tucker BJ, Bartholomay LC. Landscape, demographic, entomological, and climatic associations with human disease incidence of West Nile virus in the state of lowa, USA. Int J Health Geogr. 2008;7:19.

56. Wilke ABB, Chase C, Vasquez C, Carvajal A, Medina J, Petrie WD, et al. Urbanization creates diverse aquatic habitats for immature mosquitoes in urban areas. Sci Rep. 2019;9:15335.

57. Bowden SE, Magori K, Drake JM. Regional differences in the association between land cover and West Nile virus disease incidence in humans in the United States. Am J Trop Med Hyg. 2011;84:234-8.

58. Diuk-Wasser MA, Brown HE, Andreadis TG, Fish D. Modeling the spatial distribution of mosquito vectors for West Nile virus in Connecticut, USA. Vector Borne Zoonotic Dis. 2006;6:283-95.

59. Leisnham PT, LaDeau SL, Juliano SA. Spatial and temporal habitat segregation of mosquitoes in urban Florida. PLoS ONE. 2014;9:e91655.

60. Sallam MF, Xue R-D, Pereira RM, Koehler PG. Ecological niche modeling of mosquito vectors of West Nile virus in St. John's County, Florida, USA. Parasit Vectors. 2016;9:371

61. Botello G, Golladay S, Covich A, Blackmore M. Immature mosquitoes in agricultural wetlands of the coastal plain of Georgia, USA: effects of landscape and environmental habitat characteristics. Ecol Indic. 2013;34:304-12.

62. Burkett-Cadena N, Graham SP, Giovanetto LA. Resting environments of some Costa Rican mosquitoes. J Vector Ecol. 2013:38:12-9.

63. McPhatter LP, Debboun M. Attractiveness of botanical infusions to ovipositing Culex quinquefasciatus, Cx. nigripalpus, and $C x$. erraticus in San Antonio, Texas. J Am Assoc Mosq Contr. 2009;25:508-10.

64. Alfonzo D, Grillet ME, Liria J, Navarro J-C, Weaver SC, Barrera R. Ecological characterization of the aquatic habitats of mosquitoes (Diptera: Culicidae) in enzootic foci of Venezuelan equine encephalitis virus in western Venezuela. J Med Entomol. 2005;42:278-84.

65. O'Brien VA, Reiskind MH. Host-seeking mosquito distribution in habitat mosaics of southern Great Plains cross-timbers. J Med Entomol. 2013;50:1231-9.

66. Rey JR, Nishimura N, Wagner B, Braks MAH, Lounibos LP. Habitat segregation of mosquito arbovirus vectors in south Florida. J Med Entomol. 2007:43:1134-41.

67. Dow RP, Gerrish GM. Day-to-day change in relative humidity and the activity of Culex nigripalpus (Diptera: Culicidae). Ann Entomol Soc Am. 1970;63:995-9.

68. Shaman J, Day JF, Stieglitz MS. St. Louis encephalitis virus in wild birds during the 1990 south Florida epidemic: the importance of drought, wetting conditions, and the emergence of Culex nigripalpus (Diptera: Culicidae) to arboviral amplification and transmission. J Med Entomol. 2003;2003(40):547-54.

69. Figueroa DP, Scott S, Gonzalez CR, Veloso C, Canals M. Assessing the larval niche of Culex pipiens in Chile. Int J Mosq Res. 2016;3:18-23.

70. Burke R, Barrera R, Lewis M, Kluchinsky T, Claborn D. Septic tanks as larval habitats for the mosquitoes Aedes aegypti and Culex quinquefasciatus in Playa-Playita, Puerto Rico. Med Vet Entomol. 2010;24:117-23.

71. Calhoun LM, Avery M, Jones L, Gunarto K, King R, Roberts J, et al. Combined sewage overflows (CSO) are major urban breeding sites for Culex quinquefasciatus in Atlanta, Georgia. Am J Trop Med Hyg. 2007;77:478-84.

72. Ebel GD, Rochlin I, Longacker J, Kramer LD. Culex restuans (Diptera: Culicidae) relative abundance and vector competence for West Nile virus. J Med Entomol. 2005:42:838-43.

73. Johnson BJ, Munafo K, Shappell L, Tsipoura N, Robson M, Ehrenfeld J, et al. The roles of mosquito and bird communities on the prevalence of West Nile virus in urban wetland and residential habitats. Urban Ecosyst. 2012;15:513-31.

74. Jacob BG, Lampman RL, Ward MP, Muturi EJ, Morris JA, Caamano EX, et al. Geospatial variability in the egg raft distribution and abundance of Culex pipiens and Culex restuans in Urbana-Champaign, Illinois. Int J Remote Sens. 2009;30:2005-19.

75. Reiskind MH, Wilson ML. Culex restuans (Diptera: Culicidae) oviposition behavior determined by larval habitat quality and quantity in southeastern Michigan. J Med Entomol. 2004;41:179-86. 
76. Yee DA, Kneitel JM, Juliano SA. Environmental correlates of abundances of mosquito species and stages in discarded vehicle tires. J Med Entomol. 2010;47:53-62

77. Rochlin I, Dempsey ME, Campbell SR, Ninivaggi DV. Salt marsh as Culex salinarius larval habitat in coastal New York. J Am Assoc Mosq Contr. 2008;24:359-67.

78. Janousek TE, Olson JK. Seasonal variations in activity and size of adult females and local distribution of larvae for populations of Culex salinarius in the upper coastal zone of southeastern Texas. J Am Assoc Mosq Contr. 2006;22:47-53.

79. Lee J-H, Rowley WA. The abundance and seasonal distribution of Culex mosquitoes in lowa during 1995-97. J Am Assoc Mosq Contr. 2000;16:275-8.

80. Gingrich JB, Anderson RD, Williams GM, O'Connor L, Harkins K. Stormwater ponds, constructed wetlands, and other best management practices as potential breeding sites for West Nile virus vectors in Delaware during 2004. J Am Assoc Mosq Contr. 2006;22:282-91.

81. Andreadis TG, Armstrong PM. A two-year evaluation of elevated canopy trapping for Culex mosquitoes and West Nile virus in an operational surveillance program in the northeastern United States. J Am Assoc Mosq Contr. 2007;23:137-48.

82. Zyzak M, Loyless T, Cope S, Wooster M, Day JF. Seasonal abundance of Culex nigripalpus Theobald and Culex salinarius Coquillett in north Florida, USA. J Vector Ecol. 2002;27:155-62.

83. Chuang T-W, Hildreth MB, Vanroekel DL, Wimberly MC. Weather and land cover influences on mosquito populations in Sioux Falls, South Dakota. J Med Entomol. 2011;48:669-79.

84. Schurich JA, Kumar S, Eisen L, Moore CG. Modeling Culex tarsalis abundance on the northern Colorado front range using a landscape-level approach. J Am Assoc Mosq Contr. 2014;30:7-20.

85. Mori H, Wu J, Ibaraki M, Schwartz F. Key factors influencing the incidence of West Nile virus in Burleigh County, North Dakota. Int J Environ Res Public Health. 2018;15:1928.

86. Harrington LC, Poulson RL. Considerations for accurate identification of adult Culex restuans (Diptera: Culicidae) in field studies. J Med Entomol. 2008;45:1-8.

87. Aardema ML, vonHoldt BM, Fritz ML, Davis SR. Global evaluation of taxonomic relationships and admixture within the Culex pipiens complex of mosquitoes. Parasit Vectors. 2020;13:8.

88. Montgomery MJ, Thiemann T, Macedo P, Brown DA, Scott TW. Bloodfeeding patterns of the Culex pipiens complex in Sacramento and Yolo counties, California. J Med Entom. 2011;48:398-404

89. Ciota AT, Chin PA, Kramer LD. The effect of hybridization of Culex pipiens complex mosquitoes on transmission of West Nile virus. Parasit Vectors. 2013;6:305.

90. Hunt SK, Galatowitsch ML, McIntosh AR. Interactive effects of land use, temperature, and predators determine native and invasive mosquito distributions. Freshwater Biol. 2017;62:1564-77.
91. Ruybal JE, Kramer LD, Kilpatrick AM. Geographic variation in the response of Culex pipiens life history traits to temperature. Parasit Vectors. 2016;9:116.

92. Bingham AM, Burkett-Cadena ND, Hassan HK, Unnasch TR. Vector competence and capacity of Culex erraticus (Diptera: Culicidae) for eastern equine encephalitis virus in the southeastern United States. J Med Entomol. 2016:53:473-6.

93. Cupp EW, Tennessen KJ, Oldland WK, Hassan HK, Hill GE, Katholi CR, et al. Mosquito and arbovirus activity during 1997-2002 in a wetland in northeastern Mississippi. J Med Entomol. 2004;41:495-501.

94. Mendenhall IH, Tello SA, Neira LA, Castillo LF, Ocampo CB, Wesson DM. Host preference of the arbovirus vector Culex erraticus (Diptera: Culicidae) at Sonso Lake, Cauca Valley Department, Colombia. J Med Entomol. 2012;49:1092-102.

95. Mendenhall IH, Bahl J, Blum MJ, Wesson DM. Genetic structure of Culex erraticus populations across the Americas. J Med Entomol. 2012;49:522-34.

96. del Carpio-Orantes L, González-Clemente del MC, Lamothe-Aguilar T. Zika and its vector mosquitoes in Mexico. J Asia Pac Biodivers. 2018;11:317-9.

97. Nayar JK, Knight JW, Munstermann LE. Temporal and geographic genetic variation in Culex nigripalpus Theobald (Culicidae: Diptera), a vector of St. Louis encephalitis virus, from Florida. J Med Entomol. 2002;39:854-60.

98. Ortega-Morales A, Zavortink T, Huerta-Jiménez H, Ibáñez-Bernal S, Siller-Rodríguez Q. The mosquitoes (Diptera: Culicidae) of Hidalgo state, Mexico. Acta Trop. 2019;189:94-103.

99. Andreadis TG, Anderson JF, Vossbrinck CR. Mosquito surveillance for West Nile virus in Connecticut, 2000: isolation from Culex pipiens, Cx. restuans, Cx. salinarius, and Culiseta melanura. Emerg Infect Dis. 2001;7:670-4

100. Labarthe N, Guerrero J. Epidemiology of heartworm: what is happening in South America and Mexico? Vet Parasitol. 2005;133:149-56.

101. Rochlin I, Faraji A, Healy K, Andreadis TG. West Nile virus mosquito vectors in North America. J Med Entomol. 2019;56:1475-90.

102. Stenn T, Peck KJ, Rocha Pereira G, Burkett-Cadena ND. Vertebrate hosts of Aedes aegypti, Aedes albopictus, and Culex quinquefasciatus (Diptera: Culicidae) as potential vectors of Zika virus in Florida. J Med Entomol. 2019;56:10-7.

103. Kilpatrick AM, Kramer LD, Jones MJ, Marra PP, Daszak P. West Nile virus epidemics in North America are driven by shifts in mosquito feeding behavior. PLoS Biol. 2006;4:e82.

\section{Publisher's Note}

Springer Nature remains neutral with regard to jurisdictional claims in published maps and institutional affiliations.

Ready to submit your research? Choose BMC and benefit from:

- fast, convenient online submission

- thorough peer review by experienced researchers in your field

- rapid publication on acceptance

- support for research data, including large and complex data types

- gold Open Access which fosters wider collaboration and increased citations

- maximum visibility for your research: over $100 \mathrm{M}$ website views per year

At BMC, research is always in progress.

Learn more biomedcentral.com/submissions 